\title{
LARGE-SCALE STOCHASTIC HEREDITARY SYSTEMS UNDER MARKOVIAN STRUCTURAL PERTURBATIONS. PART I. VARIATIONAL COMPARISON THEOREMS
}

\author{
G. S. LADDE \\ Received 14 January 2005; Revised 26 October 2005; Accepted 26 October 2005
}

This three-part series work investigates the qualitative analysis of large-scale stochastic hereditary systems under random structural perturbations. The random structural perturbations are described by a Markov chain with a finite number of states. The first part of this three-part series work deals with the problem formulation and the development of mathematical tool to undertake this investigation. In this first part, we present variational comparison theorems for isolated subsystems of large-scale stochastic hereditary systems under Markovian structural perturbations. This part lays down the basis for the study of hierarchic systems.

Copyright (c) 2006 G. S. Ladde. This is an open access article distributed under the Creative Commons Attribution License, which permits unrestricted use, distribution, and reproduction in any medium, provided the original work is properly cited.

\section{Introduction}

A variety of problems that arise in the fields of engineering, medical, social, and physical sciences under hereditary and random environmental perturbations can be modeled by a system of stochastic functional differential equations [5-7, 9, 10, 19]. A feasible model for such a system is an Itô-type stochastic functional differential system perturbed by a finite-state Markov process. In this work, we propose to study the qualitative behavior of a solution of such a large-scale stochastic hereditary system.

The work is decomposed into the three parts, namely, the problem formulation and variational comparison theorems for isolated systems, the convergence and stability analysis of isolated stochastic hereditary subsystems, and large-scale stochastic hereditary systems. In this first part, we develop a very general form of well-known comparison theorems $[1-4,6,8,9,11,14-16,18,19]$.

In Section 2, the problem formulation and a description of the mathematical model are presented. For isolated subsystems, variational comparison theorems that incorporate the effects of the past history and random structural perturbations are developed in Section 3. Finally, in Section 4, examples are given to illustrate the significance of the 
presented results. The presented results extend and generalize the earlier work $[1-4,6-$ $9,11-16,18,19]$ in a systematic and unified way.

\section{Problem formulation}

Input-output systems found in the biological, physical, and social sciences can in some settings be modeled by a stochastic system that is governed by random structural dynamics of the system. In these types of systems, the knowledge about the system is limited to output information. For purposes of investigation, it is assumed that measurements $Y\left(t, x_{t}, \eta\right)$ of an unknown functional $f\left(t, x_{t}, \eta\right)$ are of the following form:

$$
Y\left(t, x_{t}, \eta\right)=f\left(t, x_{t}, \eta\right)+\sum_{r=1}^{m} \sigma_{r}\left(t, x_{t}, \eta\right) \frac{d \xi_{r}(t)}{d t}
$$

where $\sum_{r=1}^{m} \sigma_{r}\left(t, x_{t}, \eta\right)\left(d \xi_{r}(t) / d t\right)$ reflects random errors in measurement, $d \xi_{r}(t) / d t$ is Gaussian white noise, $\eta$ describes random structural dynamics of the system, and a pasthistory $x_{t}(\theta)=x(t+\theta),-\tau \leq \theta \leq 0$ reflects the hereditary effects over the time period $\tau>0$. Utilizing the continuous version of well-known stochastic approximation procedures $[12,17,20]$, a mathematical model of stochastic hereditary dynamic systems under Markovian structural perturbations is described by the following system:

$$
d x=\mathbf{F}\left(t, x_{t}, \eta(t)\right) \cdot d \boldsymbol{\xi}(t), \quad x_{t_{0}}=\varphi_{0},
$$

where $\mathbf{F}=\left(F_{0}^{T}, F_{1}^{T}, \ldots, F_{j}^{T}, \ldots, F_{m}^{T}\right)^{T}, F_{j} \in \mathscr{C}\left[R_{+} \times C^{n} \times R, \mathscr{R}^{n}\right]$ for $j \in I(0, m), t_{0} \in[0, \infty)$; $C^{n}=C\left[[-\tau, 0], R^{n}\right]$ denotes the space of continuous functions with the domain $[-\tau, 0]$ and range in $R^{n}$; $x_{t}$ is an element of $C^{n}$ defined by $x_{t}(\theta)=x(t+\theta),-\tau \leq \theta \leq 0$; for $-\tau \leq$ $\theta \leq 0, \varphi_{0}(\theta)$ is $n$-dimensional random vector defined on a complete probability space $(\Omega, \mathscr{F}, P)$ representing an initial past history of the state of the system, and it is sample continuous; $\eta=\eta(t, \omega)$ in (CHS) is a right continuous finite state $I(1, q)=\{1,2,3, \ldots, q\}$ Markov process with a constant $q \times q$ state intensity matrix $\Pi=\left(\pi_{j l}\right)$ representing random internal structural changes in the system; $\xi(t)=\left(\xi_{1}(t), \xi_{2}(t), \ldots, \xi_{m}(t)\right)^{T}$ is an $m$ dimensional normalized Wiener process with independent increments; $\eta(t)$ and $\xi(t)$ are $\mathscr{F}_{t}$-measurable for all $t \geq t_{0}$ and $\mathscr{F}_{t}$ is an increasing family of sub- $\sigma$-algebras of $\mathscr{F}_{F} ; \varphi_{0}(\theta)$, $\eta(t)$, and $\xi(t)$ are mutually independent for each $t \geq t_{0} ; \xi_{0}(t)=t, \xi(t)=\left(\xi_{0}(t), \xi_{1}(t), \xi_{2}(t)\right.$, $\left.\ldots, \xi_{m}(t)\right)$. It is assumed that $\mathbf{F}$ is smooth enough to insure the existence of a solution process $x(t)=x\left(t_{0}, \varphi_{0}\right)(t)$ of (CHS).

A system described by (CHS) may involve a large number of state variables. Using a "one shot" technique to study such a system may prove to be both analytically and computationally difficult. A more feasible approach is a decomposition-aggregation approach coupled with Lyapunov's second method. In this case, the complex stochastic hereditary system (CHS) is decomposed into smaller, simpler, and suitable $p$-interconnected subsystems (perturbed) of the following form:

$$
d x^{i}=\left[\mathbf{a}^{i}\left(t, x_{t}^{i}, \eta(t)\right)+\mathbf{c}^{i}\left(t, x_{t}, \eta(t)\right)\right] \cdot d \xi(t), \quad x_{t_{0}}^{i}=\varphi_{0}^{i},
$$


where $\mathbf{a}^{i}=\left(a_{0}^{i T}, a_{1}^{i T}, \ldots, a_{j}^{i T}, \ldots, a_{m}^{i T}\right)^{T}$ and $\mathbf{c}^{i}=\left(c_{0}^{i T}, c_{1}^{i T}, \ldots, c_{j}^{i T}, \ldots, c_{m}^{i T}\right)^{T}, a_{j}^{i} \in C\left[J \times C^{n_{i}} \times\right.$ $\left.R, R^{n_{i}}\right]$ and $c_{j}^{i} \in C\left[J \times C^{n} \times R, R^{n_{i}}\right]$ for $j \in I(0, m) ; \varphi_{0}=\left(\left(\varphi_{0}^{1}\right)^{T},\left(\varphi_{0}^{2}\right)^{T}, \ldots,\left(\varphi_{0}^{p}\right)^{T}\right)^{T}, x=$ $\left(\left(x^{1}\right)^{T},\left(x^{2}\right)^{T}, \ldots,\left(x^{p}\right)^{T}\right)^{T}$, and $\sum_{i=1}^{p} n_{i}=n$ for each $i \in I(1, p)$; the interactions (perturbations) among the $p$-subsystems of system (LHS) are described by $c^{i}$.

The qualitative analysis of each decoupled/isolated stochastic hereditary subsystem corresponding to (LHS) under Markovian structural perturbations

$$
d x^{i}=\mathbf{a}^{i}\left(t, x_{t}^{i}, \eta(t)\right) \cdot d \boldsymbol{\xi}(t), \quad x_{t_{0}}^{i}=\varphi_{0}^{i},
$$

is aggregated in Part II of this work by an energy/Lyapunov-like function for $i \in I(1, p)$. By employing an energy/Lyapunov-like function associated with each subsystem and the nature of interactions $c^{i}$ among the subsystems of (LHS) system, a very general comparison theorem is formed, and then used to study the qualitative properties of large-scale system in Part III.

Let $x(t)=\left(x^{1 T}(t), x^{2 T}(t), \ldots, x^{i T}(t), \ldots, x^{p^{T}}(t)\right)^{T}=x\left(t_{0}, \varphi_{0}\right)(t)$ and $x^{i}(t)=x^{i}\left(t_{0}, \varphi_{0}^{i}\right)(t)$ be the solution processes of large-scale system (LHS) and isolated systems (DHS) through $\left(t_{0}, \varphi_{0}\right)$ and $\left(t_{0}, \varphi_{0}^{i}\right)$, respectively.

\section{Variational comparison theorems for isolated stochastic hereditary subsystems}

Comparison theorems $[3-6,9,11-16,18,19]$ provide a very general framework for studying the qualitative properties of dynamic systems. In this section, an attempt is made to generalize and to extend an idea of variational comparison theorems $[3,8,13]$ for isolated stochastic hereditary subsystems (DHS). This result connects solution processes of stochastic hereditary subsystems with the maximal solution of comparison systems and a solution of an auxiliary systems of differential equations. As a byproduct of this, several auxiliary comparison results are formulated.

For each $i \in I(1, p)$, let us consider a following auxiliary system:

$$
\frac{d}{d t} z^{i}=f^{i}\left(t, z^{i}\right), \quad z^{i}\left(t_{0}\right)=z_{0}^{i}
$$

where $f^{i} \in C\left[J \times R^{n_{i}}, R^{n_{i}}\right]$, and it is twice continuously differentiable with respect to $z^{i}$; the solution $z^{i}\left(t, t_{0}, z_{0}^{i}\right)$ of (DAS) exists for $t \geq t_{0}$; it is unique and twice continuously differentiable with respect to the initial state $z_{0}^{i}$; and further assume that its second derivative $\left(\partial^{2} / \partial x^{i} \partial x^{i}\right) z^{i}\left(t, s, z_{0}^{i}\right)$ is locally Lipschitzian in $z_{0}^{i}$ for each $(t, s)$ and $i \in I(1, p)$.

For each $q_{i} \geq 1, V^{i} \in C\left[[-\tau, \infty) \times R^{n_{i}} \times R, R_{+}^{q_{i}}\right] ;(\partial / \partial t) V^{i}\left(t, z^{i}, y\right),(\partial / \partial x) V^{i}\left(t, z^{i}, y\right)$, and $\left(\partial^{2} / \partial x^{2}\right) V\left(t, z^{i}, y\right)$ exist and are continuous on $[-\tau, \infty) \times R^{n_{i}} \times R$; and $\left(\partial^{2} / \partial x^{2}\right) V^{i}(t$, $\left.z^{i}, y\right)$ is locally Lipschitzian in $z^{i}$ for each $(t, s), t_{0} \leq s \leq t$. We define operators $D^{+} V_{(\mathrm{DHS})}^{i}$, $D_{\mathbf{a}^{i}}^{+} V^{i}$, and $D_{\eta}^{+} V^{i}$ as follows:

$$
\begin{aligned}
D_{(\mathrm{DHS})}^{+} V^{i}\left(s, \varphi^{i}, z^{i}\left(t, s, \varphi^{i}(0)\right), \eta(s)\right) & \\
=\limsup _{h \rightarrow 0^{+}} \frac{1}{h} E[ & V^{i}\left(s+h, z^{i}\left(t, s+h, \varphi^{i}(0)+\mathbf{a}^{i}\left(s, \varphi^{i}, \eta(s)\right) \cdot \Delta \boldsymbol{\xi}(s)\right), \eta(s+h)\right) \\
& \left.\quad-V^{i}\left(s, z^{i}\left(t, s, \varphi^{i}(0)\right), \eta(s)\right) \mid F_{s}\right], \quad i \in I(1, p),
\end{aligned}
$$


4 Large-scale stochastic systems. Part I. Comparison theorems

where $\Delta \boldsymbol{\xi}(s)=\boldsymbol{\xi}(s+h)-\boldsymbol{\xi}(s)$,

$$
\begin{aligned}
& D_{\mathbf{a}^{i}}^{+} V^{i}\left(s, \varphi^{i}, z^{i}\left(t, s, \varphi^{i}(0)\right), \eta(s)\right) \\
& =\limsup _{h \rightarrow 0^{+}} \frac{1}{h} E\left[V^{i}\left(s+h, z^{i}\left(t, s+h, \varphi^{i}(0)+\mathbf{a}^{i}\left(s, \varphi^{i}, \eta(s)\right) \cdot \Delta \xi(s)\right), \eta(s)\right)\right. \\
& \left.\quad-V^{i}\left(s, z^{i}\left(t, s, \varphi^{i}(0)\right), \eta(s)\right) \mid F_{s}\right], \quad i \in I(1, p), \\
& D_{\eta}^{+} V^{i}\left(s, z^{i}\left(t, s, \varphi^{i}(0)\right), j\right) \\
& =\sum_{l=1}^{q} \pi_{j l}\left[V^{i}\left(s, z^{i}\left(t, s, \varphi^{i}(0)\right), l\right)-V^{i}\left(s, z^{i}\left(t, s, \varphi^{i}(0)\right), j\right)\right],
\end{aligned}
$$

where $\eta(s)=j$.

Remark 3.1. Under the assumptions on $f^{i}$ and $V^{i}$, we note that for $t_{0} \leq s \leq t$,

$$
D_{\mathbf{a}^{i}}^{+} V^{i}\left(s, \varphi^{i}, z^{i}\left(t, s, \varphi^{i}(0)\right), \eta(s)\right) \leq L_{i}^{D} V^{i}\left(s, \varphi^{i}, z^{i}\left(t, s, \varphi^{i}(0)\right), \eta(s)\right),
$$

where an operator $L_{i}^{D}$ is defined by

$$
\begin{aligned}
& L_{i}^{D} V^{i}\left(s, \varphi^{i}, z^{i}\left(t, s, \varphi^{i}(0)\right), \eta(s)\right) \\
&=\frac{\partial}{\partial s} V^{i}\left(s, z^{i}\left(t, s, \varphi^{i}(0)\right), \eta(s)\right)+\frac{1}{2} \operatorname{tr}\left(\frac{\partial^{2}}{\partial z_{i}^{2}} V^{i}\left(s, z^{i}\left(t, s, \varphi^{i}(0)\right), \eta(s)\right) \Theta_{i}^{D}\left(t, s, \varphi^{i}, \eta(s)\right)\right) \\
&+\frac{\partial}{\partial z^{i}} V^{i}\left(s, z^{i}\left(t, s, \varphi^{i}(0)\right), \eta(s)\right)\left[\frac{1}{2} \operatorname{tr}\left(\frac{\partial^{2}}{\partial x^{i} \partial x^{i}} z^{i}\left(t, s, \varphi^{i}(0)\right) \Lambda_{i}^{D}\left(s, \varphi^{i}, \eta(s)\right)\right)\right. \\
&\left.+\Phi^{i}\left(t, s, \varphi^{i}(0)\right) M_{i}^{D}\left(s, \varphi^{i}(0), \varphi^{i}, \eta(s)\right)\right],
\end{aligned}
$$

where

$$
\begin{gathered}
M_{i}^{D}\left(s, \varphi^{i}(0), \varphi^{i}, \eta(s)\right)=\lim _{h \rightarrow 0^{+}} \frac{1}{h} E\left[\mathbf{a}^{i}\left(s, \varphi^{i}, \eta(s)\right) \cdot \Delta \xi(s)-h f^{i}\left(s, \varphi^{i}(0)\right) \mid F_{s}\right], \\
\Lambda_{i}^{D}\left(s, \varphi^{i}, \eta(s)\right)=\lim _{h \rightarrow 0^{+}} \frac{1}{h} E\left[\left(\mathbf{a}^{i}\left(s, \varphi^{i}, \eta(s)\right) \cdot \Delta \xi(s)\right)\left(\mathbf{a}^{i}\left(s, \varphi^{i}, \eta(s)\right) \cdot \Delta \boldsymbol{\xi}(s)\right)^{T} \mid F_{s}\right],
\end{gathered}
$$

$\Theta_{i}^{D}\left(t, s, \varphi^{i}, \eta(s)\right)=\Phi^{i}\left(t, s, \varphi^{i}(0)\right) \Lambda_{i}^{D}\left(s, \varphi^{i}, \eta(s)\right) \Phi^{i T}\left(t, s, \varphi^{i}(0)\right)$, and $\Phi^{i}\left(t, s, x^{i}\right)$ is as defined in $[11,13,15,16]$.

Let us first state and provide a sketch of proof of a following auxiliary result that will be used to prove the main comparison theorem.

\section{Lemma 3.2. Assume that}

(i) $V^{i} \in C\left[[-\tau, \infty) \times R^{n_{i}} \times R, R_{+}^{q_{i}}\right] ;(\partial / \partial t) V^{i}\left(s, z^{i}, y\right),(\partial / \partial x) V^{i}\left(s, z^{i}, y\right)$, and $\left(\partial^{2} / \partial x^{2}\right) V(s$, $\left.z^{i}, y\right)$ exist and are continuous on $[-\tau, \infty) \times R^{n_{i}} \times R$; and $\left(\partial^{2} / \partial x^{2}\right) V^{i}\left(s, z^{i}, y\right)$ is locally Lipschitzian in $z^{i}$ for each $(t, s), t_{0} \leq s \leq t$; 
(ii) $z^{i}\left(t, s, z_{0}^{i}\right)$ is the solution process of auxiliary system (DAS) through $\left(s, \varphi^{i}(0)\right), t_{0} \leq$ $s \leq t$, and its second derivative $\left(\partial^{2} / \partial x^{i} \partial x^{i}\right) z^{i}\left(t, s, z_{0}^{i}\right)$ is locally Lipschitzian in $z_{0}^{i}$ for each $(t, s)$ and $i \in I(1, p)$;

(iii) $\eta$ is right-continuous finite-state Markov chain as described in (CHS). Then,

$$
\begin{aligned}
D_{(\mathrm{DHS})}^{+} & V^{i}\left(s, \varphi^{i}, z^{i}\left(t, s, \varphi^{i}(0)\right), \eta(s)\right) \\
= & L_{i}^{D} V^{i}\left(s, \varphi^{i}, z^{i}\left(t, s, \varphi^{i}(0)\right), \eta(s)\right)+D_{\eta}^{+} V\left(s, z^{i}\left(t, s, \varphi^{i}(0)\right), \eta(s)\right),
\end{aligned}
$$

where $\left(t, \varphi^{i}\right) \in R_{+} \times \mathscr{C}^{n_{i}} ; D_{(\mathrm{DHS})}^{+} V^{i}\left(s, \varphi^{i}, z^{i}\left(t, s, \varphi^{i}(0)\right), \eta(s)\right), D_{\eta}^{+} V^{i}\left(s, z^{i}\left(t, s, \varphi^{i}(0)\right), \eta(s)\right)$, and $L_{i}^{D} V^{i}\left(s, \varphi^{i}, z^{i}\left(t, s, \varphi^{i}(0)\right), \eta(s)\right)$ are as defined in (3.1), (3.2), and (3.3), respectively.

Proof. For small $h>0$, we compute

$$
\begin{aligned}
D_{(\mathrm{DHS})}^{+} V^{i}\left(s, \varphi^{i}, z^{i}\left(t, s, \varphi^{i}(0)\right), \eta(s)\right) & \\
=\limsup _{h \rightarrow 0^{+}} \frac{1}{h} E[ & V^{i}\left(s+h, z^{i}\left(t, s+h, \varphi^{i}(0)+\mathbf{a}^{i}\left(s, \varphi^{i}, \eta(s)\right) \cdot \Delta \boldsymbol{\xi}(s)\right), \eta(s+h)\right) \\
& \left.-V^{i}\left(s, z^{i}\left(t, s, \varphi^{i}(0)\right), \eta(s)\right) \mid F_{s}\right], \quad i \in I(1, p), \\
=\limsup _{h \rightarrow 0^{+}} \frac{1}{h} E[ & P\left(A_{j}\right)\left[V^{i}\left(s+h, z^{i}\left(t, s+h, \varphi^{i}(0)+\mathbf{a}^{i}\left(s, \varphi^{i}, \eta(s)\right) \cdot \Delta \boldsymbol{\xi}(s)\right), \eta(s)\right)\right. \\
\left.\quad-V^{i}\left(s, z^{i}\left(t, s, \varphi^{i}(0)\right), \eta(s)\right) \mid F_{s}\right], i \in I(1, p), & \\
& +\sum_{l \neq j}^{q} P\left(A_{j l}\right)\left[V^{i}\left(s+h, z^{i}\left(t, s+h, \varphi^{i}(0)+\mathbf{a}^{i}\left(s, \varphi^{i}, \eta(s)\right) \cdot \Delta \boldsymbol{\xi}(s)\right), l\right)\right. \\
- & \left.\left.V^{i}\left(s, z^{i}\left(t, s, \varphi^{i}(0)\right), \eta(s)\right) \mid F_{s}\right]\right], \quad i \in I(1, p),
\end{aligned}
$$

where

$$
\begin{gathered}
P\left(A_{j}\right)=P(\eta(s+h)=j \mid \eta(s)=j)=1-\pi_{j j} h+o(h), \\
P\left(A_{j l}\right)=P(\eta(s+h)=l \mid \eta(s)=j)=\pi_{j l} h+o(h), \\
\pi_{j j}=\sum_{l \neq j}^{s} \pi_{j l} .
\end{gathered}
$$

From these expressions, (3.7) can be written as

$$
\begin{aligned}
\limsup _{h \rightarrow 0^{+}} \frac{1}{h} E[ & V^{i}\left(s+h, z^{i}\left(t, s+h, \varphi^{i}(0)+\mathbf{a}^{i}\left(s, \varphi^{i}, \eta(s)\right) \cdot \Delta \boldsymbol{\xi}(s)\right), \eta(s)\right) \\
- & \left.V^{i}\left(s, z^{i}\left(t, s, \varphi^{i}(0)\right), \eta(s)\right) \mid F_{s}\right]
\end{aligned}
$$


6 Large-scale stochastic systems. Part I. Comparison theorems

$$
\begin{gathered}
+\liminf _{h \rightarrow 0^{+}} \frac{1}{h} \sum_{l \neq j}^{q} h \pi_{\eta(s) l}\left[V^{i}\left(s+h, z^{i}\left(t, s+h, \varphi^{i}(0)+\mathbf{a}^{i}\left(s, \varphi^{i}, \eta(s)\right) \cdot \Delta \boldsymbol{\xi}(s)\right), l\right)\right. \\
\left.-V^{i}\left(s, z^{i}\left(t, s, \varphi^{i}(0)\right), \eta(s)\right) \mid F_{s}\right] \\
\leq D_{(\mathrm{DHS})}^{+} V^{i}\left(s, \varphi^{i}, z^{i}\left(t, s, \varphi^{i}(0)\right), \eta(s)\right) \\
\leq \underset{h \rightarrow 0^{+}}{\limsup } \frac{1}{h} E\left[V^{i}\left(s+h, z^{i}\left(t, s+h, \varphi^{i}(0)+\mathbf{a}^{i}\left(s, \varphi^{i}, \eta(s)\right) \cdot \Delta \xi(s)\right), \eta(s)\right)\right. \\
\left.\quad-V^{i}\left(s, z^{i}\left(t, s, \varphi^{i}(0)\right), \eta(s)\right) \mid F_{s}\right] \\
+\limsup _{h \rightarrow 0^{+}} \frac{1}{h} \sum_{l \neq j}^{q} h \pi_{\eta(s) l}\left[V^{i}\left(s+h, z^{i}\left(t, s+h, \varphi^{i}(0)+\mathbf{a}^{i}\left(s, \varphi^{i}, \eta(s)\right) \cdot \Delta \xi(s)\right), l\right)\right. \\
\left.\quad-V^{i}\left(s, z^{i}\left(t, s, \varphi^{i}(0)\right), \eta(s)\right) \mid F_{s}\right] .
\end{gathered}
$$

Because of the continuity of $V^{i}$, the above expression establishes relation (3.6) in the context of the definitions of $D_{(\mathrm{DHS})}^{+} V^{i}\left(s, \varphi^{i}, z^{i}\left(t, s, \varphi^{i}(0)\right), \eta(s)\right), D_{\eta}^{+} V^{i}\left(s, z^{i}\left(t, s, \varphi^{i}(0)\right), j\right)$, and $L_{i}^{D} V^{i}\left(s, \varphi^{i}, z^{i}\left(t, s, \varphi^{i}(0)\right), \eta(s)\right)$. This completes the proof of the lemma.

Now we are ready to present a very general comparison theorem in the context of vector Lyapunov-like functions and the system of functional differential inequalities $[4,6$, $10,18]$. The presented result relates the solutions of (DHS), (DAS), and a system of comparison differential equations (CDE). We state a result with a brief sketch of its proof. The detailed proof can be formulated by utilizing the arguments in the proofs of comparison theorems for systems of deterministic functional differential equations $[4,15,16,18]$, systems of stochastic differential equations without past history [11, 13, 19], and systems of stochastic functional differential equations [1, 2, 6-9].

Theorem 3.3. Assume that

(a) $g^{i j} \in C\left[R_{+} \times R_{+}^{q_{i}} \times C_{+}^{q_{i}}, R_{+}^{q_{i}}\right], g^{i j}\left(t, u^{i}, \sigma^{i}\right)$ is concave in $\left(u^{i}, \sigma^{i}\right)$ for each $t$, quasimonotone nondecreasing in $u^{i}$ for each $\left(t, \sigma^{i}\right) \in R_{+} \times \mathscr{C}_{+}^{q_{i}}$, and increasing in $\sigma^{i}$ for each $\left(t, u^{i}\right) \in R_{+} \times R_{+}^{q_{i}}$ for each $i \in I(1, p)$ and $j \in I(1, q)$;

(b) $w^{i} \in C\left[R_{+} \times\left(R_{+}^{q_{i}}\right)^{q} \times\left(\mathscr{C}_{+}^{q_{i}}\right)^{q},\left(R^{q_{i}}\right)^{q}\right]$; it is defined by

$$
w^{i}\left(t, u^{i}, \sigma^{i}\right)=\left(w^{i 1}\left(t, u^{i}, \sigma^{i}\right)^{T}, w^{i 2}\left(t, u^{i}, \sigma^{i}\right)^{T}, \ldots, w^{i q}\left(t, u^{i}, \sigma^{i}\right)^{T}\right)^{T},
$$

where $w^{i j} \in C\left[R_{+} \times\left(R_{+}^{q_{i}}\right)^{q} \times\left(C_{+}^{q_{i}}\right)^{q}, R^{q_{i}}\right]$ and

$$
\begin{aligned}
w^{i j}\left(t, u^{i}, \sigma^{i}\right)= & \left(g_{1}^{i j}\left(t, u^{i j}, \sigma^{i j}\right)+\sum_{l=1}^{q} \lambda_{j l} u_{1}^{i l}, g_{2}^{i j}\left(t, u^{i j}, \sigma^{i j}\right)\right. \\
& \left.+\sum_{l=1}^{q} \lambda_{j l} u_{2}^{i l}, \ldots, g_{q_{i}}^{i j}\left(t, u^{i j}, \sigma^{i j}\right)+\sum_{l=1}^{q} \lambda_{j l} u_{q_{i}}^{i l}\right)^{T},
\end{aligned}
$$

where $u^{i j}, u^{i l} \in R_{+}^{q_{i}}$ for $j, l \in I(1, q)$, and the $q \times q$ matrix $\Lambda$ is defined by $\Lambda=\left(\lambda_{j l}\right)_{q \times q}$ such that $\lambda_{j l}=\pi_{j l}$ for $j \neq l$, and $\lambda_{j j}=-\pi_{j j}$ for $j=l$ for all $j, l \in I(1, q)$; 
(c) $r^{i}\left(t_{0}, \sigma_{0}^{i}\right)(t)=r^{i}(t)$ is the maximal solution of the block comparison system (BCS),

$$
\frac{d}{d t} u^{i}=w^{i}\left(t, u^{i}, u_{t}^{i}\right), \quad u_{t_{0}}^{i}=\sigma_{0}^{i}
$$

$\left(t_{0}, \sigma_{0}^{i}\right) \in R_{+} \times\left(\mathscr{C}_{+}^{q_{i}}\right)^{q}$ existing for $t \geq t_{0}$ for each $i \in I(1, p)$;

(d) hypotheses of Lemma 3.2 are satisfied, and $L_{i}^{D} V^{i}\left(s, \varphi^{i}, z^{i}\left(t, s, \varphi^{i}(0)\right), j\right)$ satisfies the following relation:

$$
L_{i}^{D} V^{i}\left(s, \varphi^{i}, z^{i}\left(t, s, \varphi^{i}(0)\right), j\right) \leq g^{i j}\left(s, V^{i}\left(s, z^{i}\left(t, s, \varphi^{i}(0)\right), j\right), V_{s}^{i}\right),
$$

where $V_{s}^{i}=V^{i}\left(s+\theta, z^{i}\left(t, s+\theta, \varphi^{i}(s+\theta)\right), j\right)$ for $\theta[-\tau, 0]$;

(e) for any solution process $x^{i}\left(t_{0}, \varphi_{0}, \eta(t)\right)(t)=x^{i}(t)$ of $(D H S), E\left[V^{i}\left(t, x^{i}(t), \eta(t)\right)\right]$ exists for $t \geq t_{0}$ and

$$
E\left[V^{i}\left(t_{0}+\theta, z^{i}\left(t, s+\theta, \varphi^{i}(\theta)\right), \eta\left(t_{0}\right)\right) \mid \eta\left(t_{0}\right)=j_{0}\right] \leq \sigma_{0}^{i j_{0}}(\theta), \quad \forall \theta \in[-\tau, 0] .
$$

Then,

$$
E\left[V^{i}\left(t, x^{i}(t), j\right) \mid x\left(t_{0}\right)=\varphi(0)\right] \leq r^{i j}\left(t_{0}, \sigma_{0}^{i}\right)(t), \quad t \geq t_{0},
$$

for all $t \geq t_{0}, i \in I(1, p)$, and $j \in I(1, q)$.

Proof. Let $x^{i}(s)=x^{i}\left(t_{0}, \varphi^{i}(0)\right)(s)$ be a solution process of (DHS) through $\left(t_{0}, \varphi^{i}(0)\right)$. We set

$$
m^{i}(s)=V^{i}\left(s, z^{i}\left(t, s, x^{i}(s)\right), \eta(s)\right), \quad \text { for } t_{0} \leq s \leq t
$$

For small $h>0$, we define

$$
m^{i j}(s+h)=E\left[V^{i}\left(s+h, z^{i}\left(t, s+h, x^{i}(s+h)\right), \eta(s+h)\right) \mid x(s)=x, \eta(s)=j\right] .
$$

We note that

$$
m^{i j}(s)=E\left[V^{i}\left(s, z^{i}\left(t, s, x^{i}(s)\right), \eta(s)\right) \mid x(s)=x, \eta(s)=j\right] .
$$

By using this notation, for each $i \in I(1, p)$, we consider

$$
\begin{aligned}
m^{i j}(s+h)- & m^{i j}(s) \\
=E[ & V^{i}\left(s+h, z^{i}\left(t, s+h, \varphi^{i}(0)+\mathbf{a}^{i}\left(s, \varphi^{i}, \eta(s)\right) \cdot \Delta \xi(s)\right), \eta(s+h)\right) \\
& \left.\quad-V^{i}\left(s, z^{i}\left(t, s, \varphi^{i}(0)\right), \eta(s)\right) \mid F_{s}\right] \\
+ & E\left[V ^ { i } \left(s+h, z^{i}\left(t, s+h, x^{i}(s+h), \eta(s+h)\right)\right.\right. \\
& \left.\left.\quad-V^{i}\left(s+h, z^{i}\left(t, s+h, \varphi^{i}(0)+\mathbf{a}^{i}\left(s, \varphi^{i}, \eta(s)\right)\right) \cdot \Delta \xi(s)\right), \eta(s+h)\right) \mid F_{s}\right],
\end{aligned}
$$

where $\varphi^{i}=x_{s}, \varphi^{i}(0)=x^{i}(s), \eta(s)=j, \Delta \xi(s)=\xi(s+h)-\xi(s), i \in I(1, p)$, and $j \in I(1, q)$. 
From this, hypotheses on $V^{i}$ and $z^{i}\left(t, s, x^{i}(s)\right)$, Lebesgue convergence theorem, and Lemma 3.2, we arrive at

$$
D^{+} m^{i j}(s) \leq L_{i}^{D} V^{i}\left(s, \varphi^{i}, z^{i}\left(t, s, \varphi^{i}(0)\right), j\right)+D_{\eta}^{+} V^{i}\left(s, z^{i}\left(t, s, \varphi^{i}(0)\right), j\right) .
$$

From (3.2), (3.12), (3.15), (3.17), (3.19), and assumptions (a) and (b), we have

$$
D^{+} m^{i}(s) \leq w^{i}\left(s, m^{i}(s), m_{s}^{i}\right), \quad \text { for } t_{0} \leq s \leq t,
$$

and from the assumption (e), we have

$$
m^{i}(\theta) \leq \sigma_{0}^{i}(\theta), \quad \theta \in[-\tau, 0] .
$$

Now, the rest of the proof can be completed by imitating the standard argument $[4,6,9$, $15,16]$.

To illustrate the scope and the significance of Theorem 3.3, we first present a corollary. Furthermore, this provides a tool to study the behavior of block comparison system (BCS).

Corollary 3.4. Assume that the hypotheses of Theorem 3.3 are satisfied. In addition, assume that $g^{i j}$ satisfies

$$
\begin{aligned}
& \sum_{k=1}^{q_{i}} d_{k} g_{k}^{i j}\left(s, V^{i}\left(s, z^{i}\left(t, s, \varphi^{i}(0)\right), j\right), V_{s}^{i}\right) \\
& \quad \leq \alpha^{i j}(s) \nu^{i}\left(s, z^{i}\left(t, s, \varphi^{i}(0)\right), j\right)+\beta^{i j}(s) \int_{-\tau}^{0} \nu^{i}\left(s+\theta, z^{i}\left(t, s+\theta, \varphi^{i}(\theta)\right), j\right) d_{\theta} \kappa^{i}(s, \theta),
\end{aligned}
$$

where $\eta(s)=j, d_{i}>0, \alpha^{i j}, \beta^{i j} \in C[J, R], \beta^{i j} \geq 0, \kappa^{i}(s, \theta)$ is a function of bounded variation and $d_{\theta} \kappa^{i}(s, \theta)$ is a nonnegative Stieltjes measure on $[-\tau, 0]$ for $t_{0} \leq s \leq t$, and

$$
\nu^{i}\left(s, z^{i}\left(t, s, \varphi^{i}(0)\right), \eta(s)\right)=\sum_{k=1}^{q_{i}} d_{k} V_{k}^{i}\left(s, z^{i}\left(t, s, \varphi^{i}(0)\right), \eta(s)\right) .
$$

Then,

$$
D_{(\mathrm{DHS})}^{+} v^{i}\left(s, \varphi^{i}, z^{i}\left(t, s, \varphi^{i}(0)\right)\right) \leq-\bar{A}^{i}(s) v^{i}\left(s, z^{i}\left(t, s, \varphi^{i}(0)\right)\right)+L^{i}\left(v_{s}^{i}\right),
$$

where $v^{i}\left(s, z^{i}\left(t, s, \varphi^{i}(0)\right)\right)=\left(\nu^{i}(t, x, 1), \nu(t, x, 2), \ldots, \nu(t, x, q)\right)^{T}$,

$$
\begin{gathered}
\bar{A}^{i}(s)=\left(a_{k l}^{i}(s)\right)_{q \times q} ; \bar{B}^{i}(s)=\operatorname{diag}\left(\beta^{i 1}(s), \beta^{i 2}(s), \ldots, \beta^{i q}(s)\right), \\
L^{i}\left(v_{s}^{i}\right)=\bar{B}^{i}(s) \int_{-\tau}^{0} v^{i}\left(s+\theta, z^{i}\left(t, s+\theta, \varphi^{i}(\theta)\right), j\right) d_{\theta} \kappa^{i}(s, \theta), \\
a_{j l}^{i}(s)= \begin{cases}\pi_{j j}-\alpha^{i j}(s), & l=j, \\
-\pi_{j l}, & l \neq j .\end{cases}
\end{gathered}
$$


Moreover,

$$
E\left[v^{i}\left(t, x^{i}(t)\right) \mid \varphi_{0}^{i}\left(t_{0}\right)=x_{0}\right] \leq r^{i}\left(t_{0}, \sigma_{0}^{i}\right)(t)
$$

whenever $v_{t_{0}}^{i} \leq \sigma_{0}^{i}$, where $r^{i}\left(t_{0}, \sigma_{0}^{i}\right)(t)$ is the maximal solution of the following linear system of functional differential equations:

$$
\frac{d}{d t} u^{i}=-\bar{A}^{i}(t) u^{i}+L^{i}\left(u_{t}^{i}\right), \quad u_{t_{0}}^{i}=\sigma_{0}^{i} .
$$

Proof. From (3.6) and the proof of Theorem 3.3, for each $j \in I(1, q)$, we have

$$
\begin{aligned}
D_{(\mathrm{DHS})}^{+} \nu^{i}(s, \varphi(0), \varphi, j) \leq & \sum_{k=1}^{q_{i}} d_{k} g_{k}^{i j}\left(s, V^{i}\left(s, z^{i}\left(t, s, \varphi^{i}(0)\right), j\right), V_{s}^{i}\right) \\
& +\sum_{l \neq j}^{q} \pi_{k l} \sum_{k=1}^{q_{i}} d_{k} V_{k}^{i}\left(s, z^{i}\left(t, s, \varphi^{i}(0)\right), l\right) \\
& -\pi_{j j} \sum_{k=1}^{q_{i}} d_{k} V_{k}^{i}\left(s, z^{i}\left(t, s, \varphi^{i}(0)\right), j\right) .
\end{aligned}
$$

Using the definition of $\nu^{i}$ and (3.22), (3.28) reduces to

$$
\begin{aligned}
D_{(\mathrm{DHS})}^{+} \nu^{i}\left(s, \varphi(0), \varphi^{i}, j\right) \leq & \sum_{k=1}^{q_{i}} d_{k}\left(\alpha^{i j}(s)-\pi_{j j}\right) V_{k}^{i}\left(s, z^{i}\left(t, s, \varphi^{i}(0)\right), j\right) \\
& +\beta^{i j}(s) \int_{-\tau}^{0} \nu^{i}\left(s+\theta, z^{i}\left(t, s+\theta, \varphi^{i}(\theta)\right), j\right) d_{\theta} \kappa^{i}(s, \theta) \\
& +\sum_{k \neq j}^{q} \pi_{j k} \nu^{i}\left(s, z^{i}\left(t, s, \varphi^{i}(0)\right), k\right) .
\end{aligned}
$$

Using the definitions of $\bar{A}^{i}(s), L^{i}\left(\nu_{s}^{i}\right), v^{i}$, and the nature of the elements of $\bar{A}^{i},(3.29)$ reduces to the desired inequality (3.24):

$$
D_{(\mathrm{DHS})}^{+} \nu^{i}\left(s, \varphi^{i}, z^{i}\left(t, s, \varphi^{i}(0)\right)\right) \leq-\bar{A}^{i}(s) v^{i}\left(s, \varphi^{i}(0)\right)+L^{i}\left(\nu_{s}^{i}\right) .
$$

The proof of inequality (3.27) follows from the uniqueness of solution of (LCS) and the application of Theorem 3.3. This completes the proof of the corollary.

Remark 3.5. If $f^{i} \equiv 0$, then Theorem 3.3 reduces to the usual comparison theorems $[2,6$, $8,9,11]$ as special cases.

\section{Examples}

In this section, a few examples are given to illustrate the scope of the results.

Example 4.1. Assume that the hypotheses of Theorem 3.3 are satisfied in the context of the following considerations: suppose that for each $i \in I(1, p), f^{i}\left(t, z_{i}\right)=A^{i}(t) z_{i}$ in (DAS), 
where $A^{i}$ is a continuous $n_{i} \times n_{i}$ matrix function. $\Phi^{i}(t, s)$ is a fundamental matrix solution of $z_{i}^{\prime}=A^{i}(t) z^{i}, \Phi^{i}(s, s)=I_{n_{i} \times n_{i}}$ (identity matrix). Further assume that $g^{i}\left(t, u^{i}, \sigma^{i}\right) \equiv 0$. In this case $r^{i}\left(t_{0}, \sigma_{0}^{i}\right)(t)=\Psi\left(t, t_{0}\right) \sigma_{0}^{i}(0)$, where $\Psi\left(t, t_{0}\right)$ is fundamental matrix solution of ordinary linear systems of differential equations corresponding to the comparison system, for each $z^{i}\left(t, s, x^{i}\right)=\Phi^{i}(t, s) x^{i}$ for each $i \in I(1, p)$. Moreover, one can choose $\sigma_{0}^{i}(\theta)=$ $V^{i}\left(t_{0}+\theta, \Phi^{i}\left(t, t_{0}+\theta\right) \varphi_{0}^{i}(\theta), \eta\left(t_{0}\right)\right)$.

Example 4.2. For $i \in I(1, p), V^{i}\left(s, z^{i}\right)=z^{i T} P^{i}(\eta(s)) z^{i}$ are admissible in Theorem 3.3 with $q_{i}=1$. Here $P_{i}$ is a nonnegative definite $n_{i} \times n_{i}$ matrix for each $i \in I(1, p)$. Further assume the conditions as described in Example 4.1. In this case, $V^{i}$ and $z^{i}\left(t, s, x^{i}\right)=\Phi^{i}(t, s) x^{i}$ are continuously differentiable with respect to $\left(s, x_{i}\right)$ for each $t, t_{0} \leq s \leq t$. In this case, $L_{i}^{D} V^{i}\left(s, \varphi^{i}, z^{i}\left(t, s, \varphi^{i}(0)\right), \eta(s)\right)$, defined in (3.3), reduces to

$$
\begin{aligned}
& L_{i}^{D} V^{i}\left(s, \varphi^{i}, z^{i}\left(t, s, \varphi^{i}(0)\right), \eta(s)\right) \\
&=\left(\Phi^{i}(t, s) \varphi^{i}(0)\right)^{T} P^{i}(\eta(s)) \Phi^{i}(t, s) M_{i}^{D}\left(s, \varphi^{i}(0), \varphi^{i}, \eta(s)\right) \\
&+\left(\Phi^{i}(t, s) M_{i}^{D}\left(s, \varphi^{i}(0), \varphi^{i}, \eta(s)\right)\right)^{T} P^{i}(\eta(s)) \Phi^{i}(t, s) \varphi^{i}(0) \\
&+\operatorname{tr}\left(P^{i}(\eta(s)) \Phi^{i}(t, s) \Lambda_{i}^{D}\left(s, \varphi^{i}, \eta(s)\right) \Phi^{i}(t, s)\right) .
\end{aligned}
$$

It is assumed that

$$
\begin{aligned}
\left(\Phi^{i}(t, s)\right. & \left.\varphi^{i}(0)\right)^{T} P^{i}(j) \Phi^{i}(t, s) M_{i}^{D}\left(s, \varphi^{i}(0), \varphi^{i}, j\right) \\
& +\left(\Phi^{i}(t, s) M_{i}^{D}\left(s, \varphi^{i}(0), \varphi^{i}, j\right)\right)^{T} P^{i}(j) \Phi^{i}(t, s) \varphi^{i}(0) \\
& +\operatorname{tr}\left(P^{i}(j) \Phi^{i}(t, s) \Lambda_{i}^{D}\left(s, \varphi^{i}, j\right) \Phi^{i}(t, s)\right) \\
\leq & \mu^{i j}(s)\left(\Phi^{i}(t, s) \varphi^{i}(0)\right)^{T} P^{i}(j) \Phi^{i}(t, s) \varphi^{i}(0) \\
& +\beta^{i j}(s) \int_{-\tau}^{0}\left(\Phi^{i}(t, s+\theta) \varphi^{i}(\theta)\right)^{T} P^{i}(j) \Phi^{i}(t, s+\theta) \varphi^{i}(\theta) d \theta .
\end{aligned}
$$

A comparison system of differential equation is constructed similar to (LCS).

Remark 4.3. Examples 4.1 and 4.2 exhibit the connection between the solution processes of (DHS), (DAS), and (BCS)/(LCS).

\section{References}

[1] M. H. Chang, G. S. Ladde, and P. T. Liu, Stability of stochastic functional differential equations, Journal of Mathematical Physics 15 (1974), no. 9, 1474-1478.

[2] G. S. Ladde, Differential inequalities and stochastic functional differential equations, Journal of Mathematical Physics 15 (1974), no. 6, 738-743.

[3] - Variational comparison theorem and perturbations of nonlinear systems, Proceedings of the American Mathematical Society 52 (1975), no. 1, 181-187.

[4] Systems of functional differential inequalities and functional differential systems, Pacific Journal of Mathematics 66 (1976), no. 1, 161-171.

[5] - Competitive processes. I. Stability of hereditary systems, Nonlinear Analysis. Theory, Methods \& Applications 1 (1977), no. 6, 607-631. 
[6] Stability of general systems in biological, physical and social sciences, Applied General Systems Research (Internat. Conf., State Univ. New York, Binghamton, NY, 1977) (G. J. Klir, ed.), NATO Conf., Ser.: Systems Sci., vol. 5, Plenum, New York, 1978, pp. 575-587.

[7] __ Competitive processes and comparison differential systems. II, Journal of Mathematical and Physical Sciences 15 (1981), no. 5, 435-454.

[8] __ Stochastic delay differential systems, Proceedings of the International Symposium on Functional Differential Equations, Kyoto, Japan, World Scientific, New Jersey, 1991, pp. 204212.

[9] Stabilizing and oscillating hereditary and random structural perturbations effects on multispecies processes, Proceedings of Conference on: Nonlinear Systems: Modelling, Simulation and Applications (S. B. Agase, ed.), Science College, Nanded, 2003, pp. 1-20.

[10] _ Qualitative Analysis of Competitive-Cooperative Process in Biological, Physical and Social Sciences, Monograph in Preparation.

[11] G. S. Ladde and V. Lakshmikantham, Random Differential Inequalities, Mathematics in Science and Engineering, vol. 150, Academic Press, New York, 1980.

[12] G. S. Ladde and B. A. Lawrence, Stability and convergence of stochastic approximation procedures under Markovian structural perturbations, Dynamic Systems and Applications 10 (2001), no. 2, $145-175$.

[13] G. S. Ladde and M. Sambandham, Stochastic Versus Deterministic Systems of Differential Equations, Monographs and Textbooks in Pure and Applied Mathematics, vol. 260, Marcel Dekker, New York, 2004.

[14] G. S. Ladde and D. D. Šiljak, Connective stability of large-scale stochastic systems, International Journal of Systems Science 6 (1975), no. 8, 713-721.

[15] V. Lakshmikantham and S. Leela, Differential and Integral Inequalities: Theory and Applications. Vol. I: Ordinary Differential Equations, Academic Press, New York, 1969.

[16] _ Differential and Integral Inequalities: Theory and Applications. Vol. II: Functional, Partial, Abstract, and Complex Differential Equations, Academic Press, New York, 1969.

[17] M. B. Nevel'son and R. Z. Has'minskiľ, Stochastic Approximation and Recursive Estimation, Translations of Mathematical Monographs, vol. 47, American Mathematical Society, Rhode Island, 1973.

[18] G. R. Shendge, Finite systems of functional differential inequalities and minimax solutions, Acta Mathematica Academiae Scientiarum Hungaricae 25 (1974), 21-30.

[19] D. D. Šiljak, Large-Scale Dynamic Systems. Stability and Structure, North-Holland, New York, 1978.

[20] M. T. Wasan, Stochastic Approximation, Cambridge Tracts in Mathematics and Mathematical Physics, no. 58, Cambridge University Press, London, 1969.

G. S. Ladde: Department of Mathematics, The University of Texas at Arlington, Arlington,

TX 76019, USA

E-mail address: ladde@uta.edu 


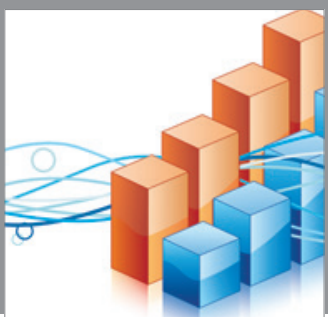

Advances in

Operations Research

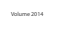

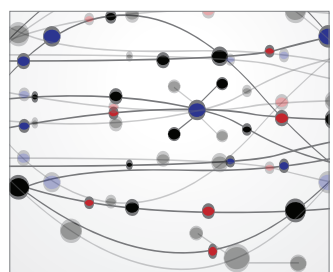

\section{The Scientific} World Journal
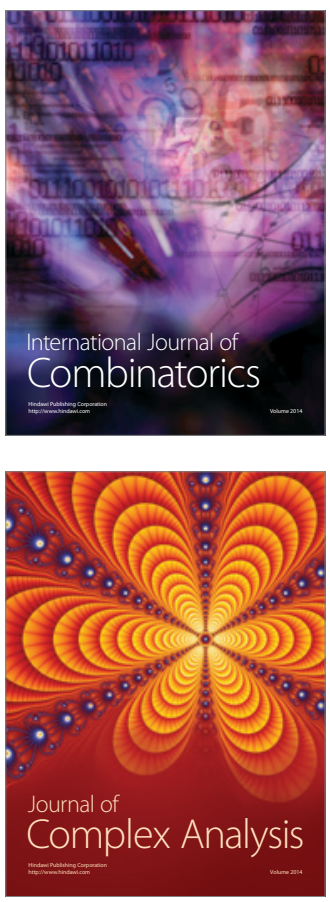

International Journal of

Mathematics and

Mathematical

Sciences
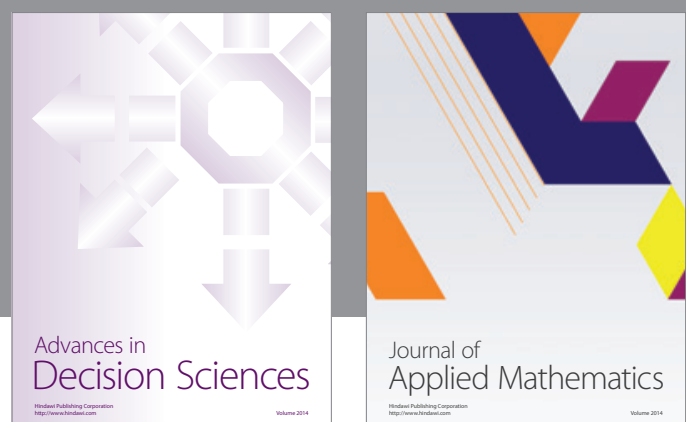

Journal of

Applied Mathematics
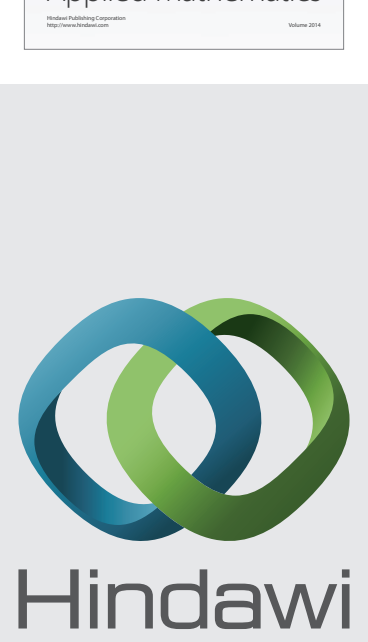

Submit your manuscripts at http://www.hindawi.com
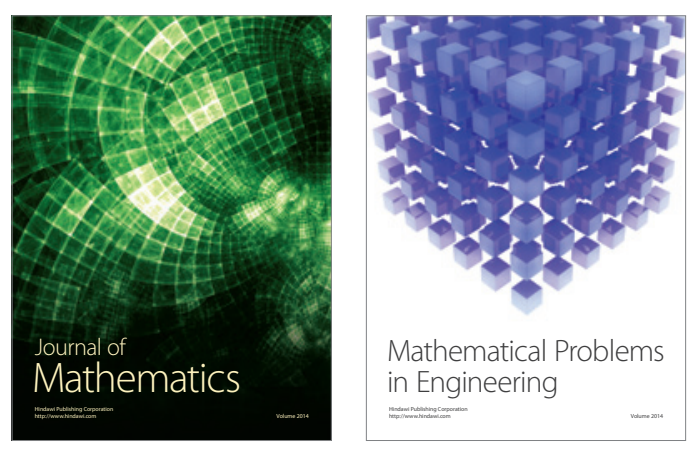

Mathematical Problems in Engineering
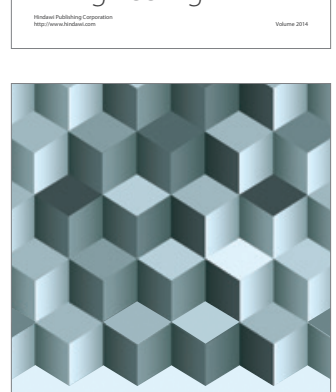

Journal of

Function Spaces
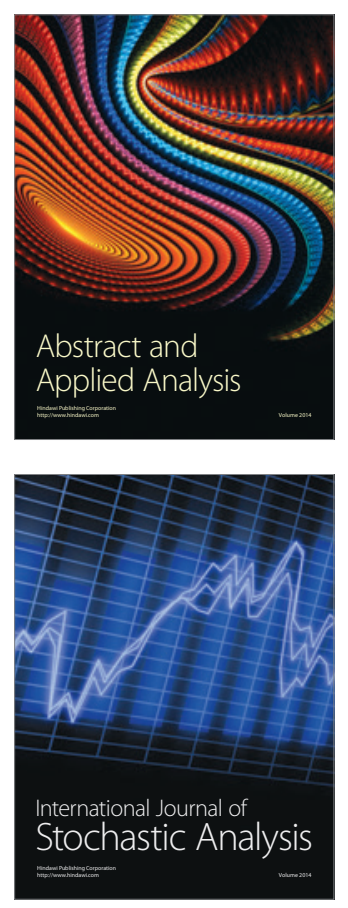

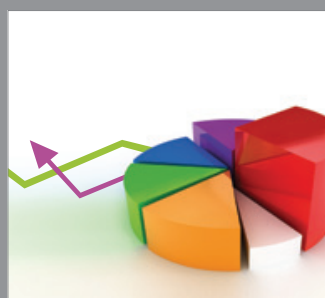

ournal of

Probability and Statistics

Promensencen
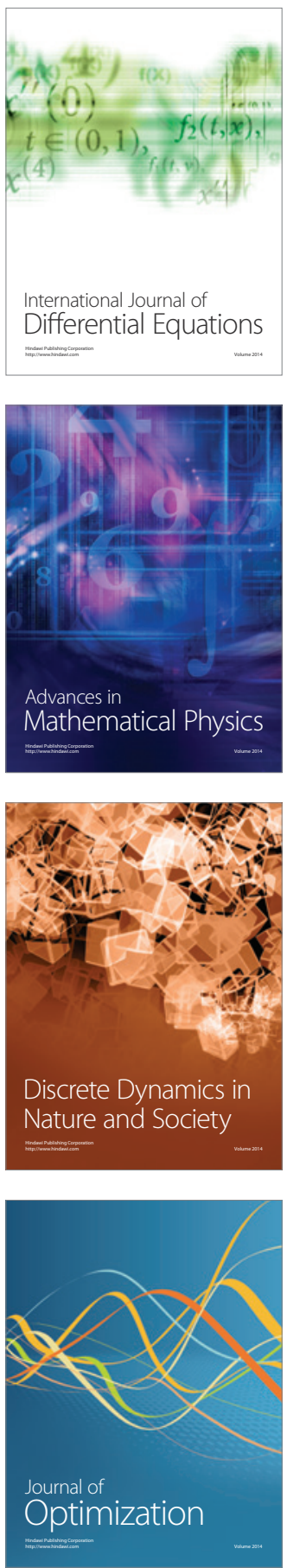